\title{
Avaliação do uso de realidade aumentada e gamificação para o treinamento de habilidades em laparoscopia
}

\author{
Julian Valerioo $^{1,4}$, Luís Fernando Silva ${ }^{1,4}$, Alysson Santos ${ }^{2,4}$, Antônio Melo Junior ${ }^{2,4}$, \\ Fernando Trinta $^{1,4}$, Antônio Aldo Melo Filho ${ }^{3,4}$ e Windson Viana ${ }^{2,4}$ \\ ${ }^{1}$ Grupo de Redes de Computadores, Engenheria de Software e Sistemas - Fortaleza - CE \\ ${ }^{2}$ Instituto UFC Virtual -Fortaleza - CE \\ ${ }^{3}$ Departamento de Cirúrgia - Escola de Medicina - Fortaleza - CE \\ ${ }^{4}$ Universidade Federal do Ceará - Fortaleza - CE \\ \{julian, luissantos\}@great.ufc.br, \{alysson, melojr\}@virtual.ufc.br \\ fernandotrinta@great.ufc.br, aamelo@ufc.br, windson@great.ufc.br
}

\begin{abstract}
Minimally Invasive Surgeries (MIS) require learning and training of non-conventional skills of surgeons (e.g., hand-eye coordination, manual dexterity and precision). In laparoscopic box-trainers, these skills can be practiced with simple navigation and coordination activities. In this paper, we propose to extend laparoscopic box-trainers with Augmented Reality (AR) and Gamification techniques. Our proposal introduces an extra camera, and uses tracking algorithms in order to monitor movements of the manipulated objects. With this approach, we are able to observe the user performance during the training activity (e.g., time to finish the activity, errors). We designed and developed two activities for navigation skills training. An initial user evaluation conducted with a group of eighteen users was implemented. Results indicate a good usability acceptance of the box-trainer. We also observed a significant difference in performance between surgeons and students of the first year of residency.
\end{abstract}

Resumo. Cirurgias minimamente invasivas requerem aprendizagem e treinamento de habilidades não-convencionais de cirurgiões (por exemplo, coordenação mão-olho, destreza manual e precisão). Os processos de ensino e aprendizagem envolvem em geral o uso de caixas-pretas simuladoras para o treinamento dessas habilidades que podem ser praticadas com atividades de navegação com as pinças e de coordenação de movimentos simples. Neste trabalho, propomos estender caixas-pretas de treinamento laparoscópico com Realidade Aumentada (RA) e técnicas de gamificação. A proposta apresenta uma câmera adicional e utiliza algoritmos de rastreamento de objetos a fim de controlar os movimentos dos objetos manipulados. O simulador é capaz de observar o desempenho do aprendiz durante a atividade de formação (por exemplo, tempo para terminar a atividade, erros). Foi implementada uma avaliação inicial de usabilidade com um grupo de dezoito usuários. Os resultados indicam uma boa aceitação da proposta. Foi observada também uma diferença significativa no desempenho entre os cirurgiões e os alunos do primeiro ano de residência, um indicativo positivo para a sua utilização no processo de ensinoaprendizagem de habilidades em MIS. 


\section{Introdução}

A cirurgia laparoscópica ou cirurgia minimamente invasiva (do inglês, Minimally Invasive Surgery - MIS), diferentemente da cirurgia convencional, consiste na abertura de pequenos orifícios no local da cirurgia (e.g. abdômen, joelho) e inserção de uma câmera iluminada e também de instrumentos cirúrgicos para realização do procedimento. Como os cirurgiões operam através do vídeo capturado pela câmera exibido em monitores, novas habilidades motoras e cognitivas são necessárias para o procedimento. As técnicas laparoscópicas exigem habilidades complexas, em que o cirurgião tem que executar precisamente a translação, rotação ou manipulações de objetos, entre outros movimentos específicos [Vassiliou et al. 2006]. Existem vários obstáculos enfrentados pelos professores no processo de ensino-aprendizagem de habilidades manuais para MIS, tais como: $(i)$ a falta de simulações adequadas de rigidez e elasticidade dos tecidos humanos, (ii) alto custo dos equipamentos de treinamento, (iii) questões legais e éticas de algumas técnicas de ensino-aprendizagem (utilização de animais e cadáveres) e (iv) a subjetividade presente ao avaliar este tipo de atividade.

Uma das ferramentas mais comuns para treinar habilidades em MIS são as caixaspretas laparoscópicas, também chamadas de caixas simuladoras para treinamento de videolaparoscopia (do inglês, Laparocospic Box-trainers). Essas caixas têm dimensões semelhantes a um tórax humano inflado ou a um abdômen, e estão equipadas com câmeras conectadas a uma tela de computador [Vassiliou et al. 2006]. As caixas pretas são úteis na fase inicial do treinamento em MIS, quando as habilidades motoras devem ser aprendidas e dominadas pelos alunos [Khine et al. 2011]. As vantagens deste método de treinamento são a presença de feedback sensorial para o aluno e o baixo custo financeiro quando comparado a outros métodos de treinamento. No entanto, caixas-pretas convencionais não são capazes de simular um procedimento de cirurgia (por exemplo, colecistectomia) e não fornecem avaliação automatizada sobre o desempenho do aluno. Uma abordagem mais recente é exatamente a de incluir Realidade Aumentada (RA) nas caixas pretas simuladoras de videolaparoscopia [Lin et al. 2014, Lahanas et al. 2014]. Realidade Aumentada, neste caso, é um conjunto de informações gráficas que são geradas por um computador a partir da visão do aprendiz no interior da caixa simuladora (i.e., o vídeo provido pela câmera interna da caixa). Este tipo de treinamento é, em geral, mais caro do que as caixas convencionais, pois requer equipamentos específicos, como câmeras extras, vidros, sensores, etc. Não obstante, as técnicas de RA permitem a avaliação objetiva do aprendiz e melhoram a imersão e a interação sem aumento excessivo nos custos se comparado a simuladores com dispositivos hápticos e realidade virtual.

A proposta do presente trabalho é pesquisar possibilidades de extensão de simuladores caixa preta com técnicas de Realidade Aumentada (RA) e o uso de gamificação (do inglês, Gamification). Este último termo define o uso de elementos de jogos (por exemplo, classificação, pontuação e recompensa) em contextos distintos de jogos digitais e analógicos. O ambiente proposto é composto de um simulador físico, software de atividades gamificadas com Realidade Aumentada e dois cenários das atividades (i.e., objetos reais manufaturadas e/ou prototipados em impressoras 3D). O simulador é chamado de LARG (Laparoscopic Augmented Reality Games) e oferece práticas voltadas para aquisição de habilidades de navegação, coordenação mão-olho e percepção de profundidade. Objetos 3D são inseridos no ambiente com RA a fim de melhorar a imersão do aprendiz durante as práticas. Pontuação, efeitos sonoros, e um ranking são alguns objetos 
gamificados que foram incluídos nas atividades. O objetivo é tanto aumentar a motivação e engajamento dos alunos quanto fornecer aos professores uma ferramenta para avaliação do aprendiz.

\section{LARG - Laparoscopic Augmented Reality Games}

A pesquisa apresentada neste trabalho foi concebida através de uma equipe multidisciplinar que inclui profissionais da área de educação em cirurgia médica, designer de jogos e cientistas da Computação. Em um primeiro esforço de pesquisa, uma caixa preta foi construída a partir de uma estrutura plástica modificada para abrigar câmeras. Uma atividade de RA similar ao jogo Genius foi desenvolvida e apresentada em [Carvalho et al. 2013]. Esse primeiro modelo de simulador serviu de base para a criação da arquitetura atual do LARG, que será apresentada a seguir.

\subsection{A Arquitetura do LARG}

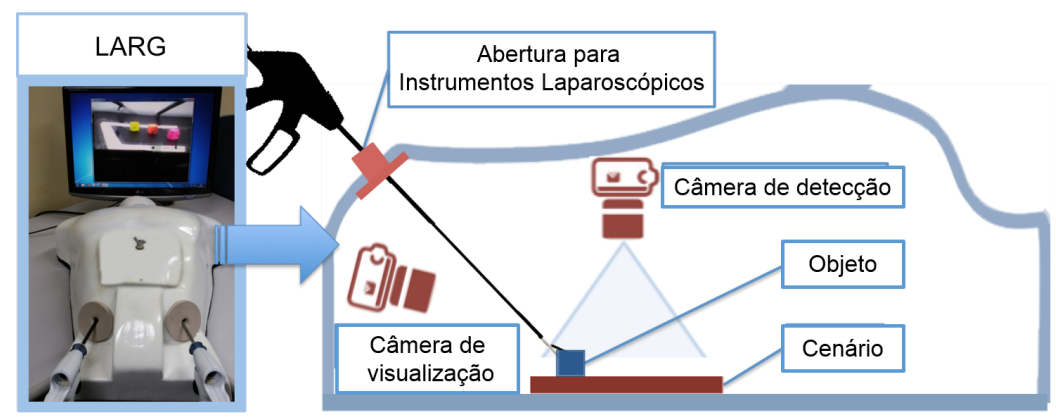

Figura 1. Disposição dos componentes físicos do LARG.

Após a construção do primeiro protótipo, resolveu-se criar uma nova solução de hardware e de software. O LARG é atualmente composto por um software gamificado, uma caixa de fibra de vidro que imita um abdômen humano, duas câmeras, instrumentos laparoscópicos convencionais (pinça de apreensão), e cenas do jogo das atividades de treinamento. As duas câmeras e cenários das atividades são colocados dentro da caixa, enquanto os instrumentos são introduzidos através das aberturas, conforme ilustrado na Figura 1.Um cenário, que consiste numa estrutura rígida de plástico e de borracha, é colocado numa posição previamente estabelecida. A câmera de monitoramento é fixa no teto da caixa, e suas imagens são usadas para monitorar mudanças no cenário, como a movimentação de objetos ou a posição das pinças. A câmera captura imagens da cena que são mescladas com as projeções de RA a serem exibidas ao usuário. O software que compõe a nova versão do LARG foi desenvolvido com a OGRE 3D para renderizar os cenários virtuais e a interface gráfica, e usa a OpenCV para realizar o reconhecimento de padrões, detecção de objetos e de movimento.

\subsection{Princípios de Design do novo LARG}

Os princípios de design norteadores desta pesquisa são: (i) criar um simulador de baixo custo financeiro; (ii) prover feedback sensorial para os aprendizes com a mescla de Realidade Aumentada e objetos reais e (iii) gamificar as atividades de treinamento; e, por fim, (iv) fornecer uma avaliação do desempenho dos aprendizes. 
Para manter a vantagem de baixo custo financeiro das caixas-pretas convencionais, a solução LARG adiciona apenas uma câmera extra. Esta câmera acompanha o movimento de objetos reais manipulados pelos instrumentos de laparoscopia e o processamento de suas imagens é que provê as bases computacionais para a avaliação do desempenho dos aprendizes. Os objetos manipulados foram concebidos para serem manuseados pelo aprendiz, são feitos de plástico ou de borracha, de modo a proporcionar rigidez e elasticidade. Na primeira versão do LARG, havia a suposição de que a Realidade Aumentada poderia substituir a maioria dos objetos das atividades, entretanto, observou-se que estes são fundamentais para fornecer ofeedback sensorial exigido para a aprendizagem das habilidades.

O uso de gamificação em softwares educacionais e jogos criados com esse objetivo (jogos sérios e edugames) tem proporcionado novas abordagens de interação, bem como atividades mais atraentes para os aprendizes [Clua 2014]. Pesquisadores já mostraram também ganhos efetivos de jogos digitais no treinamento de habilidades laparoscópicas. De fato, pesquisas conduzidas por [Jalink et al. 2015] já comprovam que usuários previamente treinados com base em um protótipo de jogo Nintendo Wii ${ }^{\mathrm{TM}}$ apresentaram melhores resultados do que usuários que foram treinados apenas nas caixas laparoscópicas. Com uma abordagem de gamificação, nosso objetivo é tornar as atividades de treinamento mais envolventes. No simulador LARG, as tarefas de formação são adaptadas em níveis de jogo, tempo, avisos sonoros, pontuação e recompensas, que são utilizados para melhorar a experiência de aprendizagem.

\section{Atividades gamificadas de treinamento de habilidades em MIS}

Um dos objetivos desta pesquisa é projetar um conjunto de atividades para treinamento de habilidades em navegação, percepção de profundidade e coordenação mão-olho semelhantes às atividades do programa MISTELS (The McGill Inanimate System for Training and Evaluation of Laparoscopic Skills). Esse programa de treinamento é utilizado nos processos de ensino-aprendizagem em residências de cirurgia e têm demonstrado ser um instrumento válido e confiável para ensinar habilidades laparoscópicas básicas [Vassiliou et al. 2006].

Nessa segunda versão da pesquisa foram criadas duas tarefas. Ambas exploram coordenação mão-olho, destreza manual, precisão, e percepção de profundidade com o uso dos instrumentos laparoscópicos. A primeira tarefa é chamada de Repita-o-Caminho e foca na introdução ao uso do simulador com uma atividade mais simples, voltada para a aprendizagem da navegação com instrumentos laparoscópicos (i.e., mover objetos seguindo um caminho). Já a segunda tarefa, chamada de PEG Transfer (transferência de borracha), é um pouco mais complexa e consiste na transferência de um conjunto de pequenas borrachas entre pinos de uma base rígida. A metodologia utilizada para desenvolver as atividades foi realizada em cinco etapas. Primeiramente, foram criadas a malha 3D (relativa à atividade) e as animações a serem exibidas com o uso de Realidade Aumentada. Em seguida, a malha foi enviada para uma impressora 3D ou foi manufaturada, o que produziu uma réplica física de cada cenário (Etapa 2). Na Etapa 3, o modelo 3D é importado para o software do LARG, para que o programador possa controlar animações e definir as regras do jogo. Na Etapa 4, os algoritmos de visão computacional são selecionados e configurados para controlar os dispositivos manipulados (por exemplo, uma borracha vermelha). As atividades e os algoritmos de detecção são, então, interligados 
concebendo o jogo executado pelo aprendiz (Etapa 5). A Figura 2 mostra essas cinco etapas, desde a descrição física do cenário até a exibição da tarefa gamificada.

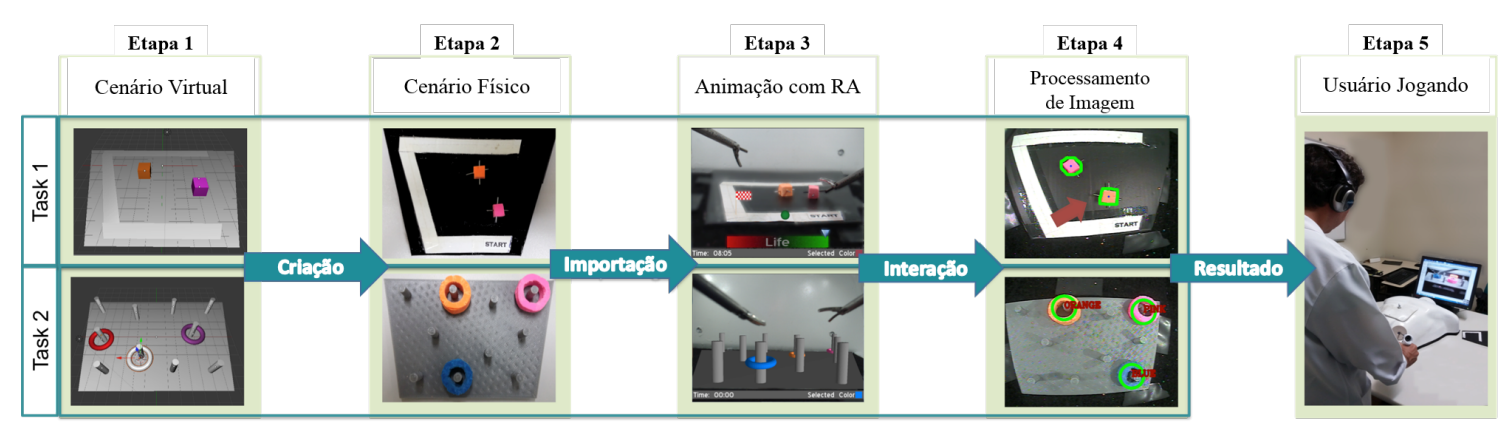

Figura 2. Fluxo de Desenvolvimento da Atividade Gamificada.

\subsection{Repita-o-Caminho}

A tarefa 1, Repita-o-Caminho, foi produzida baseada em estudos e discussões entre os autores do artigo, acerca do que seria relevante para o treinamento de habilidades básicas no simulador. A tarefa começa com a apresentação de uma animação que indica ao usuário o caminho que ele deve seguir com os instrumentos laparoscópicos. O sistema seleciona aleatoriamente um elemento do cenário, e apresenta a escolha para o usuário (por exemplo, o objeto laranja). A cena mostra também um começo e um ponto de extremidade, juntamente com um percurso não linear que os interconecta. O elemento tem de ser conduzido através do caminho, enquanto o simulador apresenta indicações visuais usando RA. Efeitos sonoros alertam cada vez que o usuário passa dos limites do percurso. Após o objeto alcançar o ponto final, a tarefa termina mostrando a pontuação do usuário (baseado em critérios como o número de vezes que o jogador desviou do caminho proposto). A Figura 2 apresenta screenshots da atividade (Tarefa 1).

\subsection{PEG-Transfer}

A tarefa 2, a transferência de borracha (PEG-Transfer), é uma versão modificada da atividade do mesmo nome existente no programa MISTELS. Ela começa com o software do LARG detectando as posições das borrachas do cenário, e aleatoriamente selecionando uma coluna disponível. Em seguida é apresentada uma animação em 3D da borracha selecionada sendo deslocada da sua posição atual para a coluna selecionada. Assim, o aprendiz tem de efetuar o movimento indicado pela animação para completar a tarefa adequadamente. Enquanto isso, um algoritmo de processamento de imagem verifica se a borracha é colocada na coluna correta. Depois que o sistema detecta se a borracha selecionada foi movida corretamente, é selecionada aleatoriamente outra borracha e uma nova coluna. Essa seleção acontece até que todas as três borrachas na cena tenham sido movidas, ou caso o limite de 120 segundos de tempo venha a expirar. Finalmente, a tarefa apresenta a pontuação alcançada, que é calculada com base no tempo que o aprendiz levou para completar a tarefa. A Figura 3 apresenta screenshots da atividade. Na parte esquerda da figura é exibida a Realidade Aumentada que indica qual atividade deve ser feita (no caso, mover a borracha rosa). A parte direita da figura exibe a visão do jogo logo após o fim da animação de R.A., na qual o aprendiz dá início a execução da tarefa e dispõe ainda de 1 minuto e 18 segundos para terminá-la. Um vídeo ilustrando a execução do PEG Transfer está disponível em https : / / youtu . be/DTurtiRBRq4. 
CBIE-LACLO 2015

Anais do XXVI Simpósio Brasileiro de Informática na Educação (SBIE 2015)
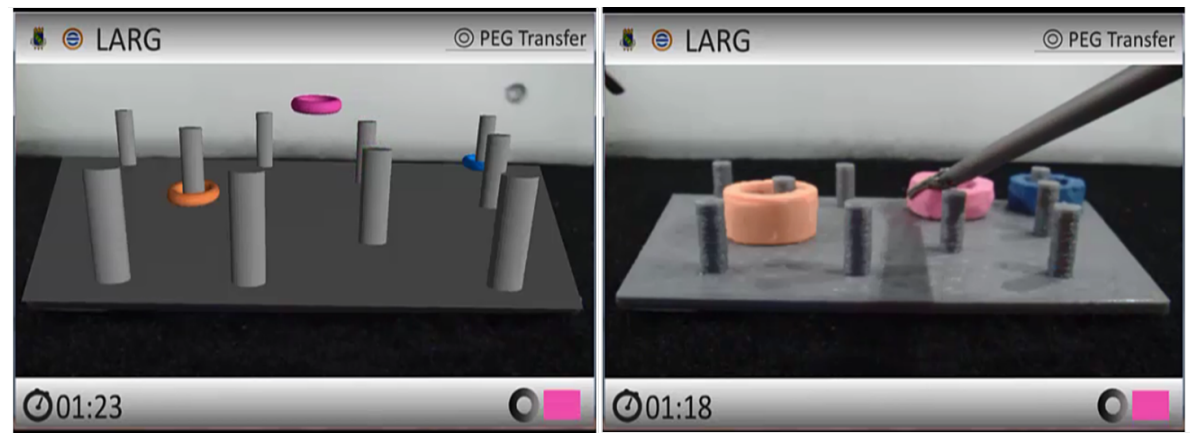

Figura 3. PEG Transfer. a) Realidade Aumentada indicando a tarefa e b) aprendiz executando a atividade no mundo "real".

\section{Avaliação}

Depois de projetar e desenvolver as atividades gamificadas, foi implementada uma avaliação de usabilidade para o LARG. Os principais objetivos da avaliação foram $(i)$ detectar o potencial de sua eficácia na formação de competências laparoscópicas e seu consequente uso no processo de ensino-aprendizagem, e (ii) medir a usabilidade do sistema e a sua jogabilidade. A solução foi avaliada por cirurgiões e estudantes de medicina com diferentes níveis de experiência em dois hospitais. Os usuários foram classificados em dois grupos: Especialistas, composto por cirurgiões ou médicos no período final da residência em cirurgia (ou seja, aqueles que já têm formação laparoscópica e mais de uma dezena de cirurgias realizadas); e Novatos, composto por estudantes e médicos de graduação no período inicial da residência em cirurgia (ou seja, aqueles sem ou com um mínimo de experiência em cirurgia laparoscópica). Durante a avaliação, 10 especialistas e 8 usuários novatos realizaram ambas as atividades três vezes, totalizando 54 ensaios para cada atividade. Essa estratégia de separação em grupos conforme o nível de expertise visa avaliar se o simulador é capaz de discernir, de forma significativa, quem já possui a habilidade/conhecimento a ser treinado. Neste caso, o esperado é que os especialistas devam ter performance melhores, em média, do que os aprendizes. Além disso, deve-se procurar avaliar se a cada nova interação existe uma melhora da habilidade, já que isto é um indicativo de que o aluno está aprendendo a técnica exigida [Lahanas et al. 2014].

\subsection{Materiais e Instrumentos}

Antes de utilizar o simulador, os usuários responderam a um primeiro conjunto de perguntas sobre seus antecedentes em matéria de formação laparoscópica e seu perfil profissional, informando a sua residência ou o nível de estágio, sua experiência anterior em MIS usando a caixas de treinamento, simuladores com RA ou RV, e se eles participaram de algum curso ou treinamento de procedimentos médicos assistidos por computador. O segundo conjunto de questões foi elaborado a partir das recomendações propostas por Savi et al na montagem de instrumentos de avaliação de jogos educacionais [Savi et al. 2010]. Ele destina-se a fornecer dados sobre cada atividade de forma independente, buscando assim, informações sobre as técnicas de gamificação empregadas, o design, o nível de dificuldade, se o usuário notou melhorias em suas habilidades ao longo das atividades, e sua motivação para a formação e aprendizagem utilizando o ferramenta. Finalmente, os usuários puderam emitir sugestões para versões futuras. 
CBIE-LACLO 2015

Anais do XXVI Simpósio Brasileiro de Informática na Educação (SBIE 2015)

\subsection{Procedimentos}

Antes de iniciar a avaliação, os usuários receberam orientações sobre o simulador. Eles realizaram cada atividade três vezes. A primeira atividade pediu que o usuário movesse um objeto específico da cena e arrastasse-o através de um caminho exibido por uma animação. Usuários começavam com pontuação de 100, e os pontos eram descontados quando o objeto deixava a trajetória indicada. A segunda atividade exigiu mais perícia dos usuários, uma vez que o usuário tinha que, dentro de dois minutos, mover três borrachas de suas posições originais para lugares aleatórios. Nesta atividade, a pontuação é inversamente proporcional ao tempo necessário para terminá-la.

\subsection{Resultados e Discussão}

Na Figura 4 (Part A) apresentamos os resultados das pontuações obtidas pelos usuários em cada uma das três tentativas(E1, E2, E3) realizadas na primeira tarefa. Podemos observar nos dois grupos que conforme os usuários repetiam a tarefa, as pontuações melhoravam, aproximando-se. Todos os usuários obtiveram melhor pontuação na terceira tentativa do que na primeira, mostrando que os usuários melhoraram seu desempenho conforme repetiam a tarefa, principalmente os aprendizes. Os resultados também mostraram que usuários especialistas obtiveram pontuações maiores e com menor variação. A média de pontos obtidos nos grupos Exp(Especialistas) e Nov(Novatos) foi de 76,84 e 67,67 pontos respectivamente, entretanto as distribuições nos dois grupos não apresentou diferenças significativas (Mann-Whitney $\mathrm{U}=29, \mathrm{n} 1=10, \mathrm{n} 2=8, \mathrm{P}<0.18$ two-tailed). Este resultado ocorreu devido ao fato que dois aprendizes obtiveram pontuações bem acima da média, inclusive com um deles atingindo a melhor pontuação das atividades. Uma possível explicação para obtenção de desempenho tão satisfatório deve-se ao fato de ambos possuírem o hábito de jogar em dispositivos eletrônicos, e de um deles ter utilizado um simulador laparoscópico anteriormente, conforme indicado no questionário de avaliação. Apesar de a primeira tarefa apresentar menor disparidade entre as pontuações obtidas nos dois grupos, o tempo necessário para a conclusão da atividade revelou-se consideravelmente menor entre os especialistas, conforme mostrado na Figura 4 (Part B). O

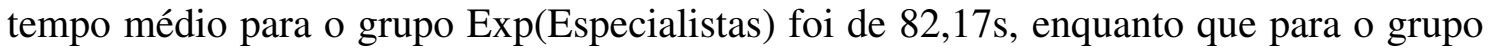
Nov(novatos) foi de 144,34s, sendo assim, a distribuição entre os dois grupos apresentou diferença significativa (Mann-Whitney $\mathrm{U}=72, \mathrm{n} 1=10, \mathrm{n} 2=8, \mathrm{P}<0.01$ two-tailed). Desta forma, podemos concluir que apesar de o grupo composto por usuários especialistas realizar as atividades mais rapidamente, sua precisão não foi muito superior a do grupo de aprendizes.

Os testes realizados na segunda tarefa apresentaram maior disparidade nos resultados obtidos entre especialistas e aprendizes. Analisando-se as médias, pôde-se observar que o grupo Exp(Especialistas) obteve 56,34 pontos enquanto o grupo Nov(novatos) obteve 30,34 pontos. Além disso, ao realizar testes de significância, observamos haver diferenças significativas entre o desempenho dos dois grupos nesta atividade (MannWhitney $\mathrm{U}=11, \mathrm{n} 1=10, \mathrm{n} 2=8, \mathrm{P}<0.01$ two-tailed). A pontuação foi obtida subtraindo o tempo necessário para finalizar a tarefa de 120, ou seja, usuários que não concluíssem a atividade em menos de dois minutos receberiam pontuação zero. A maioria dos aprendizes obteve baixas pontuações durante a primeira e segunda tentativas, seguido por uma melhora na última execução. Vários especialistas atingiram altas pontuações, com baixa variação entre as repetições, conforme exibido na Figura 4 (Part C). 
A Figura 5 apresenta uma seleção dos resultados mais relevantes obtidos através das seguintes indagações presentes no questionário de avaliação:

- Q4- Me senti estimulado a aprender e treinar habilidades com o jogo;

- Q7- Após concluir o jogo pude notar melhorias nas habilidades que pratiquei durante a realização das atividades;

- Q8- O nível de dificuldade da tarefa 1 e 2 estava ideal para o aprendizado;

- Q11- A atividade me permitiu treinar adequadamente manipulação de objetos e estimativa de profundidade;

- Q13- As técnicas de gamificação adotadas me motivaram a executar as atividades;

- Q14- O feedback (sonoro, visual, tátil) durante a realização das atividades estava ideal;

- Q15- A execução das atividades foi relevante para o aprendizado de habilidades básicas para a realização de cirurgia laparoscópica;
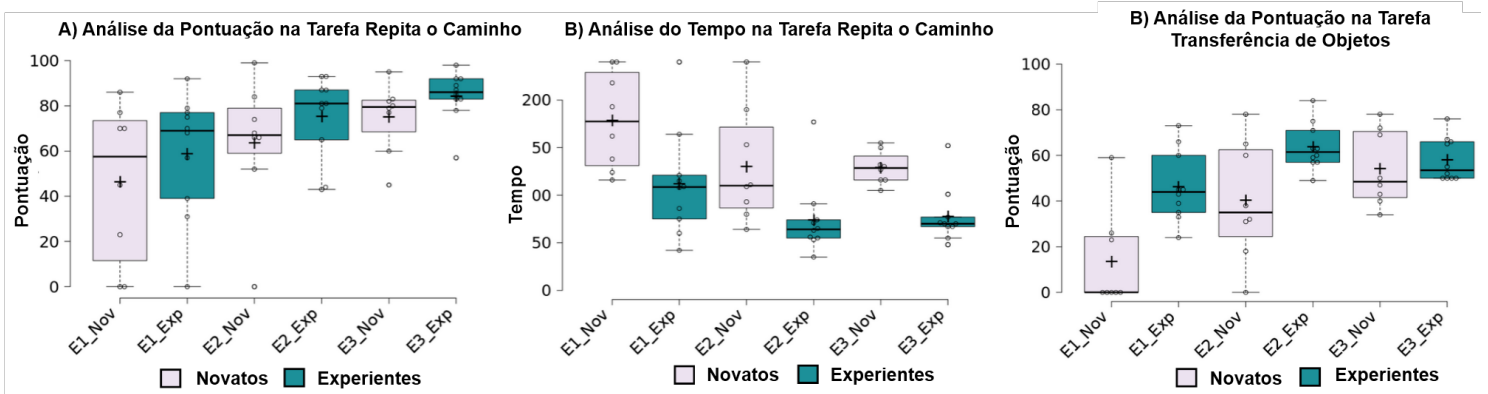

Figura 4. Box plots comparando os dois grupos em cada teste (E1, E2, E3). O gráfico (A) mostra a pontuação do usuário (100 - erros de percurso) na tarefa Siga-o-Caminho, enquanto o gráfico (B) mostra o tempo utilizado pelo usuário para concluí-la. $O$ gráfico (C) apresenta a pontuação obtida pelo usuário (120 tempo para finalizar) para a tarefa Peg Transfer.

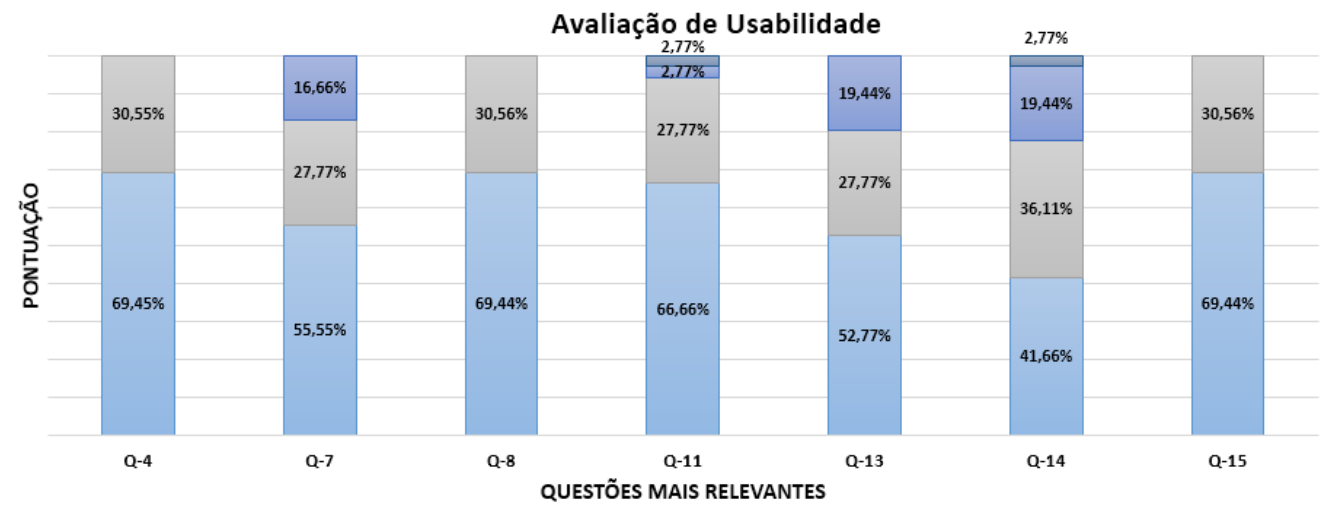

$\square$ Concordo Totalmente $\square$ Concordo Parcialmente $\square$ Indiferente $\square$ Discordo Parcialmente $\square$ Discordo Totalmente

Figura 5. Resultados mais relevantes obtidos com a avaliação de usabilidade.

Para realizar a análise dos dados da avaliação, utilizamos quatro das cinco alternativas presentes na escala Likert (concordo totalmente, concordo parcialmente, discordo parcialmente e discordo totalmente) divididas em duas categorias: concordo e discordo. As respostas indiferentes foram descartadas na análise a seguir. Os resultados obtidos 
com o teste destacaram que a maioria dos usuários se sentiu motivado a utilizar o LARG, além disso, todos os usuários informaram que o jogo apresentava um nível de dificuldade apropriado para o aprendizado. Cerca de $95 \%$ dos usuários afirmaram que as tarefas propostas permitiram a eles exercitar adequadamente atividades básicas de MIS. Outros 95\% asseguraram ter melhorado suas habilidades ao longo do treinamento, confirmando assim os resultados obtidos através da análise de desempenho. Com respeito às técnicas de gamificação, 80,5\% dos usuários avaliaram-nas como ótimas, enquanto 2,7\% reportaram que ofeedback do jogo(som, gráfico, retorno tátil) estava insatisfatório, especialmente os efeitos sonoros. Por fim, todos os usuários asseguraram que o treinamento foi importante para a melhoria no aprendizado de habilidades básicas para MIS.

\section{Trabalhos Relacionados}

Estudos comprovam que o uso de caixas simuladoras, mesmo as mais simples, traz benefícios para o aprendizado e treinamento de habilidades básicas para a realização de MIS [Beard et al. 2014]. O uso de técnicas de RA nesses simuladores oferece ainda a avaliação objetiva do aprendiz e melhora a imersão e a interação durante o treinamento das habilidades. Uma das primeira propostas que utiliza RA é o ProMIS-AR [Van Sickle et al. 2005]. O ProMIS-AR utiliza três câmeras, uma localizada no lado direito e outra no lado esquerdo do manequim que capturam os movimentos do usuário, e uma terceira câmera que fica localizada na região inferior do abdômen sendo a principal do sistema, por onde o usuário visualiza a tarefa que está executando.

Mais recentemente, Lahanas et al. (2014) desenvolveram um simulador composto por três tarefas, a fim de treinar a percepção de profundidade, a coordenação mão-olho e a ambidestria [Lahanas et al. 2014]. RA é usada para mostrar objetos 3D das tarefas de treinamento. Os usuários têm que usar instrumentos laparoscópicos reais para realizá-las corretamente. Os sensores feitos por medida são adicionados aos instrumentos laparoscópicos, a fim de permitir a interação com os elementos de realidade virtual. A desvantagem dessa abordagem é que os elementos manipulados não são reais, o que limita o feedback sensorial e a força percebida pelos usuários durante o treinamento. Em um trabalho semelhante, Lin et al (2014) propôs o uso de RA e objetos reais em duas tarefas de treinamento de habilidades. Uma câmera 3D adicional e um conjunto de sensores ópticos são adicionados à caixa simuladora para reconhecer o movimento de objetos reais. O simulador fornece feedback sensorial mais elevado do que a abordagem anterior, e também mede o desempenho dos utilizadores. No entanto, os objetos virtuais não são inseridos na visão do mundo real, o que diminui a imersão [Lin et al. 2014].

Confrontando o trabalho desenvolvido nessa pesquisa com os trabalhos citados, pode-se apontar como principais vantagens do LARG o uso das técnicas de gamificação, Realidade Aumentada com objetos e cenários reais. Essa combinação trouxe vantagens significativas para o treinamento e aprendizado. Além disso, a abordagem permite $f e-$ edback háptico real em conjunto com a avaliação objetiva do usuário, o que permite ao aprendiz, e ao instrutor, verificar se está fazendo a tarefa corretamente.

\section{Conclusão e Trabalhos Futuros}

Neste trabalho, foi apresentado e avaliado o LARG, uma ferramenta gamificada que utiliza Realidade Aumentada para melhorar habilidades básicas de MIS. Os resultados obtidos através das avaliações com usuários mostraram boa aceitação do simulador e seu 
potencial de uso nos processos de ensino-aprendizagem das residências em cirurgia, portanto concluímos que o simulador pode ser utilizado como complemento para realizar o treinamento de habilidades básicas para a realização de cirurgia minimamente invasiva. Como trabalho futuro, atividades mais complexas, como corte e sutura, além de práticas que explorem ambidestria serão concebidas. O intuito é o desenvolvimento de um programa completo gamificado para o treinamento de habilidades laparoscópicas. Além disso, deseja-se ampliar as avaliações de usabilidade e impacto cognitivo aumentando a quantidade de usuários e o tempo de aprendizagem.

\section{Agradecimentos}

Este trabalho foi apoiado parcialmente pelo CNPq (MCT / CNPq 14/2013 - Universal), sob número de concessão 484.255 / 2013-4.

\section{Referências}

Beard, J. H., Akoko, L., Mwanga, A., Mkony, C., and O’Sullivan, P. (2014). Manual laparoscopic skills development using a low-cost trainer box in tanzania. Journal of surgical education, 71(1):85-90.

Carvalho, W. V. d., Melo Junior, A. J. M., Junior, G. M., de Souza Júnior, H. C., and Melo Filho, A. A. (2013). Inserção de técnicas de gamificação e realidade aumentada para auxílio no ensino de medicina.

Clua, E. W. G. (2014). Jogos sérios aplicados a saúde. Journal of Health Informatics, 6.

Jalink, M. B., Goris, J., Heineman, E., Pierie, J.-P. E., and Henk, O. (2015). Face validity of a wii u video game for training basic laparoscopic skills. The American Journal of Surgery.

Khine, M., Leung, E., Morran, C., and Muthukumarasamy, G. (2011). Homemade laparoscopic simulators for surgical trainees. The clinical teacher, 8(2):118-121.

Lahanas, V., Loukas, C., Smailis, N., and Georgiou, E. (2014). A novel augmented reality simulator for skills assessment in minimal invasive surgery. Surgical endoscopy, pages $1-11$.

Lin, Y., Luo, Y., Lee, C., Yang, S., and Yu, D. (2014). A pc-based laparoscopic surgery skills training and assessment system. In Engineering in Medicine and Biology Society (EMBC), 2014 36th Annual International Conference of the IEEE, pages 498-501. IEEE.

Savi, R., Von Wangenheim, C. G., Ulbricht, V., and Vanzin, T. (2010). Proposta de um modelo de avaliação de jogos educacionais. RENOTE, 8(3).

Van Sickle, K., McClusky III, D., Gallagher, A., and Smith, C. (2005). Construct validation of the promis simulator using a novel laparoscopic suturing task. Surgical Endoscopy and Other Interventional Techniques, 19(9):1227-1231.

Vassiliou, M., Ghitulescu, G., Feldman, L., Stanbridge, D., Leffondre, K., Sigman, H., and Fried, G. (2006). The mistels program to measure technical skill in laparoscopic surgery. Surgical Endoscopy And Other Interventional Techniques, 20(5):744-747. 